\title{
Die Erweiterung von Konzepten und Definitionen von Suchtverhalten
}

\author{
Joël Billieux Claus Vögele \\ Institute for Health and Behaviour, University of Luxembourg, Esch-sur-Alzette, Luxembourg
}

Substanzgebrauchsstörungen («substance use disorders»; SUD) stellen weltweit eine herausragende Bedrohung für die öffentliche Gesundheit dar. Im Jahr 2016 war Alkoholmissbrauch global für 3 Millionen Todesfälle (5,3\% aller Todesfälle) und für 132,6 Millionen durch Behinderung gekennzeichnete Lebensjahre («disabilityadjusted life years»; DALYs) verantwortlich $(5,1 \%$ der DALYs weltweit) [WHO, 2018]. Tabakkonsum ist verantwortlich für mehr als 7 Millionen Tote pro Jahr [WHO, 2017]. Zusammengenommen zählen Alkohol- und Tabakkonsum zu den Hauptursachen vermeidbarer Todesfälle. Vor allem in den USA, aber auch in europäischen Ländern, ist die Zahl der betäubungsmittelabhängigen Menschen sprunghaft angestiegen - eine Entwicklung, die vor allem durch neue synthetische Opioide begünstigt wird [Karila et al., 2018]. Zusätzlich zu Alkohol und Tabak tragen psychotrope Substanzen (z.B. Heroin, «Crack»-Kokain, Metamphetamin) zunehmend zu dem großen gesundheitlichen Schaden, der durch Drogenmissbrauch entsteht, bei [Nutt et al., 2010]. Weitere Erkenntnisse zur Ätiologie und zum Verlauf von SUD sind somit dringend vonnöten, um Präventions- und Therapiemaßnahmen zu verbessern und diese an das sich ständig vergrößernde Arsenal von psychotropen Substanzen (z.B. «Designerdrogen») und neue Abhängigkeitsverhaltensweisen (z.B. «chemsex», d.h. Drogenmissbrauch zur Steigerung sexueller Erfahrungen) anzupassen.

Abhängigkeitsstörungen sind nicht mehr auf SUD beschränkt. Obwohl Verhaltenssüchte (d.h. substanzungebundene Suchterkrankungen) seit Jahrzehnten untersucht werden (Abb. 1) [Marks, 1990], fand erst 2013 ein Paradigmenwechsel statt, als die American Psychiatric Association (APA) zum ersten Mal die Glücksspielstörung als diagnostische Kategorie in das DSM-5 [APA, 2013] aufnahm (zuvor war die Störung als "pathologisches Spielen» unter den Impulskontrollstörungen subsumiert; DSM-5 = fünfte Auflage des Diagnostic and Statistical Manual of Mental Disorders). Diese neue Konzeptualisierung der Glücksspielstörung wird durch eine umfangreiche Evidenz zu Ähnlichkeiten mit SUD gestützt, wie vergleichbare neurobiologische und kognitive Ein- schränkungen und gemeinsame psychosoziale und genetische Risikofaktoren [Potenza, 2006]. Zusätzlich zur Glücksspielstörung sind in den vergangenen 20 Jahren weitere Verhaltenssüchte untersucht worden, die durch neue Technologien vermittelt werden (z.B. unkontrollierter Gebrauch von Internetdiensten wie Videospielen, Cybersex oder sozialen Netzwerken) [Kuss und Billieux, 2017]. Die Weltgesundheitsorganisation (WHO) veröffentlichte 2015 einen Bericht, demzufolge der exzessive Gebrauch von Informationsund Kommunikationstechnologien (ICTs) ein ernstzunehmendes Problem für die öffentliche Gesundheit darstellt [WHO, 2015]. Dabei kommt Online-Videospielen eine besondere Rolle zu: Umfangreiche klinische und epidemiologische Studien belegen, dass exzessives Videospielen zu psychischen Belastungen und funktionalen Einschränkungen führen kann [Stein et al., 2018]. Die kürzliche Aufnahme von Videospielsucht als psychische Störung in die 11. Version der Internationalen Statistischen Klassifikation der Krankheiten und verwandter Gesundheitsprobleme (International Statistical Classification of Diseases and Related Health Problems, 11th Edition; ICD-11) hat eine weitreichende Bedeutung. Hiermit wurde - 5 Jahre nachdem Glücksspielsucht im DSM-5 SUD gleichgestellt worden war - die Videospielsucht als zweite psychische Störung als Verhaltenssucht anerkannt. Obwohl sie derzeit noch nicht in den nosologischen Klassifikationssystemen (ICD, DSM) als eigenständige Störungen anerkannt sind, werden auch andere Verhaltensauffälligkeiten wie pathologisches Kaufen, pathologisches Essverhalten und die suchtartige Nutzung von Social Media oder von Online-Pornografie als Abhängigkeiten konzeptualisiert und behandelt [Brand et al., 2018].

Nach der Veröffentlichung des DSM-5 und der Anerkennung des Konzepts von Verhaltenssucht ist allerdings festzustellen, dass in einer stetig wachsenden Anzahl von Studien ein breites Spektrum von Alltags- und Freizeitaktivitäten (z.B. Tanzen, Arbeiten, Selfies schießen) als mögliche Verhaltenssüchte klassifiziert wird. Angesichts dieser Proliferation von möglichen neuen Störungen haben einige Autoren vor der Pathologisierung und der damit einhergehen-

\section{KARGER}

() 2018 S. Karger GmbH, Freiburg

Fax +497614520714 


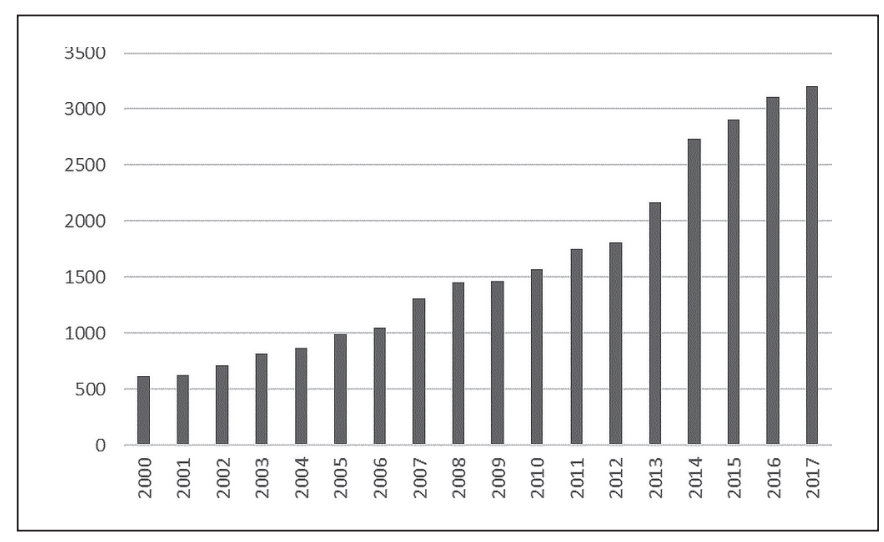

Abb. 1. Veröffentlichungen zu Verhaltenssüchten zwischen 2000 und 2017. Die Suche wurde am 21. Oktober 2018 in PubMed durchgeführt. Alle berücksichtigten Artikel erwähnen entweder «behavioral addiction» oder «behavioural addiction» als Schlüsselwörter. 2232 Publikationen aus dem Jahr 2018 waren bereits verfügbar, wobei diese nicht in die Abbildung eingeflossen sind.

den Stigmatisierung von weitverbreiteten Verhaltensweisen gewarnt [Billieux et al., 2015] und eine stärkere Differenzierung in der Diagnose dieser Störungen gefordert, um das intensive, oft mit Leidenschaft ausgeübte, aber unproblematische Verhalten von pathologischem und deswegen behandlungsbedürftigem Verhalten zu unterscheiden [Kardefelt-Winther et al., 2017]. Nötig sind deswegen neue Forschungsergebnisse zur Ätiologie von Verhaltenssüchten, um Präventions- und Behandlungsstrategien zu entwickeln, aber auch um Grenzen bei deren Konzeptualisierung aufzuzeigen. Beispielsweise müssen die unterscheidenden Merkmale einer behandlungsbedürftigen Sucht und gesundem, leidenschaftlichem Verhalten, dessen Ausübung als befriedigend und erfüllend erlebt wird, geklärt werden.

Wir sind vor dem Hintergrund dieser Entwicklungen überzeugt, dass dieses Themenheft zu «Sucht» aktuell und informativ für die Leserschaft der VERHALTENSTHERAPIE ist. Unser Ziel ist es, Sie mit den neuesten Entwicklungen hinsichtlich der Konzeptualisierung, Diagnostik und Behandlung von Süchten bekannt zu machen, unabhängig davon, ob diese Süchte mit dem Konsum von psychotropen Substanzen verbunden sind oder nicht. Wir haben dazu empirische und konzeptuelle Beiträge von deutschsprachigen Kolleginnen und Kollegen eingeladen, die sich mit SUD oder Verhaltenssüchten befassen. Sechs Artikel finden sich im vorliegenden Themenheft, darunter 3 Originalarbeiten zu SUD [Pätz et al., 2018; Schawohl und Odenwald, 2018; Schünemann et al., 2018] und 3 konzeptuelle Beiträge zu Verhaltenssüchten [Laskowski et al., 2018; Müller et al., 2018; Wegmann und Brand, 2018].

Pätz et al. [2018] untersuchten die Wirkung einer kognitiv-behavioralen Gruppentherapie zur Raucherentwöhnung in einer Stichprobe von alkoholabhängigen Patienten. Obwohl statistisch nicht signifikant, war bei der Interventionsgruppe zum Therapieende und zum Follow-up der Anteil der abstinenten Patienten größer als bei der Kontrollgruppe (ohne kognitive Verhaltenstherapie). Im Beitrag von Schawohl und Odenwald [2018] werden Ergebnisse zur Vorhersage des Therapieabbruchs anhand einer Gruppe von Patienten mit psychischen Störungen, die in einer
Hochschulambulanz behandelt wurden, vorgestellt. Unter einer ganzen Reihe von Prädiktoren, einschließlich soziodemografischer und klinischer Variablen (z.B. Krankheitsschwere), erklärte ein riskanter Alkoholkonsum zu Beginn der Therapie einen signifikanten Anteil der Therapieabbrüche. Schünemann et al. [2018] widmen sich in ihrer Untersuchung den Fragen, ob eine suchtspezifische kognitive Partnerschaftsintervention unter stationären Versorgungsbedingungen mit Veränderungen in der Partnerschaftszufriedenheit und der psychischen Belastung assoziiert ist und welche Patienten besonders von der Partnerschaftsintervention profitieren. Die Ergebnisse zeigen allerdings, dass Partnerschaftsinterventionen im subjektiven Erleben der Patienten unter Versorgungsbedingungen kurzfristig keinen zusätzlichen positiven Effekt haben.

Unter den 3 konzeptuellen Beiträgen zu Verhaltenssüchten bieten Müller et al. [2018] eine aktuelle Übersichtsarbeit zur Diagnostik und Behandlung von internetbezogenen Störungen, wobei der Schwerpunkt auf der Darstellung von verhaltenstherapeutischen Behandlungsstrategien liegt. Die Autoren kommen zu dem Schluss, dass in der Therapie ein besonderes Augenmerk auf die Etablierung einer ausreichenden Veränderungsmotivation gelegt werden sollte, um bestehende motivationale Defizite und Ambivalenzen der Patienten aufzufangen. Wegmann und Brand [2018] diskutieren in ihrer Arbeit, inwiefern zentrale Konstrukte aus der Forschung zu substanzgebundener Sucht (z.B. Reizreaktivität und Craving) auf Verhaltenssüchte übertragbar sind. Aus ihren Erkenntnissen leiten die Autoren Empfehlungen für therapeutische Konzepte ab, die vor allem Reizreaktivität und Craving in den Fokus rücken. Laskowski et al. [2018] empfehlen auf der Grundlage ihrer aktuellen Übersicht zu pathologischem Kaufverhalten die nosologische Einordnung von pathologischem Kaufen als Kaufsucht und damit als Verhaltenssucht (wie dies bereits im ICD-11 für Videospielsucht der Fall ist).

Zusammen betrachtet demonstrieren die Beiträge dieses Themenhefts die klinische Bedeutung der untersuchten Fragen, jedoch auch die Unterschiede zwischen SUD und Verhaltenssüchten, z.B. was ihren jeweiligen Entwicklungsstand der Konzepte und ihren Forschungsstand betrifft. Während in den Artikeln zu SUD Originaldaten aus klinischen Arbeiten, einschließlich Evaluations- und kontrollierten Wirksamkeitsstudien, berichtet werden, sind die Beiträge zu Verhaltenssüchten konzeptuellen Fragen gewidmet. Obwohl die Ähnlichkeiten zwischen SUD und Verhaltenssüchten überwiegen, hat die Forschung im Bereich der Verhaltenssüchte noch einen langen Weg vor sich, um denselben Fortschritt wie für SUD zu erreichen und somit empirisch validierte Präventions- und Therapieansätze zu ermöglichen. Wir sind sicher, dass dieses Themenheft dazu einen Beitrag leistet, indem die Beiträge eine aktuelle Übersicht zum Forschungsstand beider Bereiche bieten. Wir hoffen, dass die Inhalte dieser Ausgabe zu mehr Forschung zu SUD und Verhaltenssüchten anregen.

\section{Disclosure Statement}

Die Autoren erhielten keine finanzielle oder andere Unterstützung für diese Arbeit und erklären, dass sie keinen Interessenskonflikt haben.
Billieux/Vögele 


\section{Literatur}

APA (American Psychiatric Association): Diagnostic and Statistical Manual of Mental Disorders: DSM-5, ed 5. Arlington, VA, American Psychiatric Association, 2013

Billieux J, Schimmenti A, Khazaal Y: Are we overpathologizing everyday life? A tenable blueprint for behavioral addiction research. J Behav Addict 2015;4:119-123.

Brand M, Billieux J, Demetrovics Z, et al: What disorders should be considered as «other specified disorders due to addictive behaviours»? J Behav Addict 2018;7(Suppl 1):46.

Kardefelt-Winther D, Heeren A, Schimmenti A, et al: How can we conceptualize behavioral addiction without pathologizing common behaviors? Addiction 2017; 112:1709-1715.

Karila L, Marillier M, Chaumette B, et al: New synthetic opioids: part of a new addiction landscape. Neurosci Biobehav Rev 2018;pii: S0149-7634(18)30114-3.

Kuss DJ, Billieux J: Technological addictions: conceptualisation, measurement, etiology and treatment. Addict Behav 2017;64:231-233.

Laskowski NM, Trotzke P, Müller A: Brauchen versus kaufen: Wenn Warenkonsum zur Sucht wird. Verhaltenstherapie 2018;28:DOI: 10.1159/000493888.
Marks I: Behavioural (non-chemical) addictions. Brit J Addict 1990;85:1389-1394.

Müller KW, Scherer L, Beutel ME, Wölfling K: Verhaltenstherapeutische Ansätze bei internetbezogenen Störungen: Diagnostik, Motivation und Verhaltensmodifikation. Verhaltenstherapie 2018;28:DOI: 10.1159/ 000494460.

Nutt DJ, King LA, Phillips LD; Independent Scientific Committee on Drugs: Drug harms in the UK: a multicriteria decision analysis. Lancet 2010;376:1558-1565.

Pätz T, Frischknecht U, Dinter C, et al: Tabakentwöhnung mit kognitiv-behavioraler Gruppentherapie während einer 3-wöchigen qualifizierten Alkoholentzugsbehandlung: Effekte auf den Tabakkonsum. Verhaltenstherapie 2018;28:DOI: 10.1159/000492087.

Potenza MN: Should addictive disorders include non-substance-related conditions? Addiction 2006;101:142151.

Schawohl A, Odenwald M: Häufigkeit und Risikofaktoren des Therapieabbruchs in einer verhaltenstherapeutischen Hochschulambulanz: Der Einfluss von riskantem Alkoholkonsum zu Therapiebeginn. Verhaltenstherapie 2018;28:DOI: 10.1159/000492086. chünemann O, Lindenmeyer J, Heinrichs N: Effekte einer suchtspezifischen Partnerschaftsintervention in einer stationären Entwöhnungsbehandlung. Verhaltenstherapie 2018;28:DOI: 10.1159/000487770.

Stein DJ, Billieux J, Bowden-Jones $\mathrm{H}$, et al: Balancing validity, utility and public health considerations in disorders due to addictive behaviours. World Psychiatry 2018;17:363-364.

Wegmann E, Brand M: Reizreaktivität und Craving bei Verhaltenssüchten mit Fokus auf Internetnutzungsstörungen. Verhaltenstherapie 2018;28:DOI: $10.1159 / 000493918$.

WHO (World Health Organization): Report on Public Health Implications of Excessive Use of the Internet, Computers, Smartphones and Similar Electronic Devices. Geneva, World Health Organization, 2015.

WHO (World Health Organization): Report on the Global Tobacco Epidemic, 2017: Monitoring Tobacco Use and Prevention Policies. Geneva, World Health Organization, 2017

WHO (World Health Organization): Global Status Report on Alcohol and Health 2018. Geneva, World Health Organization, 2018.

\section{Impressum}

ISSN Print Edition: 1016-6262

ISSN Online Edition: 1423-0402

Journal Homepage: http://www.karger.com/ver

Publication Data: Volume 28, 2018 of 'Verhaltenstherapie' appears with 4 issues.

Copyright: $\odot 2018$ by S. Karger Verlag für Medizin und Naturwissenschaften GmbH, Freiburg (Germany). All rights reserved. No part of this publication may be translated into other languages, reproduced or utilized in any form or by any means, electronic or mechanical, including photocopying, recording, microcopying, or by any information storage and retrieval system, without permission in writing from the publisher.

Disclaimer: The statements, opinions and data contained in this publication are solely those of the individual authors and contributors and not of the publisher and the editor(s). The appearance of advertisements in the journal is not a warranty, endorsement, or approval of the products or services advertised or of their effectiveness, quality or safety. The publisher and the editor(s) disclaim responsibility for any injury to persons or property resulting form any ideas, methods, instructions or products referred to in the content or advertisements.

Subscription Rates: Subscriptions run for a full calendar year. Prices are given per year.

Print:

Online:

EUR 163.00 + postage and handling

EUR 163.00

Combined (print+online): $\quad$ EUR $213.00+$ postage and handling

Postage and handling (added to print and print+online):

EUR 13.00 (Germany), EUR 19.00 (Rest of World)

Discount subscription prices:

Please enquire about reduced rates for members of related societies.

Back Volumes and Single Issues: Information on availability and prices of single print issues and print or electronic back volumes can be obtained from Customer Service at aboservice@karger.com

Die Erweiterung von Konzepten und Definitionen von Suchtverhalten
For customers in Germany: Please contact your local bookstore or S. Karger Verlag für Medizin und Naturwissenschaften $\mathrm{GmbH}$ Wilhelmstr. 20A, 79098 Freiburg (Germany) Tel. +49761 452070 , Fax +497614520714 aboservice@karger.com

For customers in all other countries: Please contact your bookshop or S. Karger AG

Allschwilerstr. 10, 4009 Basel (Switzerland)

Tel. +416130611 11, Fax +41613061234

karger@karger.com

Advertising: Correspondence should be addressed to the publisher.

S. Karger Verlag für Medizin und Naturwissenschaften $\mathrm{GmbH}$

Attn. Ellen Zimmermann (Head of Marketing)

e.zimmermann@karger.com

Price list No. 23 of January 1, 2018 is effective.

Publisher: S. Karger Verlag für Medizin und Naturwissenschaften GmbH

Wilhelmstr. 20A, 79098 Freiburg (Germany)

www.karger.de, information@karger.com

V.i.S.d.P. (Person responsible according to the German Press Law):

Susanne Meister

Type setting: S. Karger AG, Basel

Printing: PRINTEC OFFSET medienhaus, 34123 Kassel, Germany.

Printed on acid-free and non-aging paper (ISO 9706).

ISBN 978-3-318-06460-5

e-ISBN 978-3-318-06461-2

\section{Indexing/Abstracting}

Listed in bibliographic services, including:

Web of Science / Social Sciences Citation Index / Science Citation Index Expanded /

Current Contents - Social \& Behavioral Science / Google Scholar / PsycINFO / Scopus /

Embase / Pathway Studio / EMCare / WorldCat

Beilagenhinweis: Diese Ausgabe enthält eine Beilage des Hogrefe Verlags, Göttingen. 